\title{
Dermoid of the Nasopharynx Causing Neonatal Respiratory Distress
}

\author{
Denise Manica $^{1,2}$ Cátia Saleh Neto ${ }^{1,2}$ Cláudia Schweiger ${ }^{1,2}$ Marcelo Cortina ${ }^{1}$ Gabriel Kuhl1,2 \\ ${ }^{1}$ Department of Otolaryngology, Hospital de Clínicas de Porto Alegre, \\ Porto Alegre/RS, Brazil \\ 2 Universidade Federal do Rio Grande do Sul, Porto Alegre/RS, Brazil

\begin{abstract}
Address for correspondence Denise Manica, MD, MSc, Avenida João Pessoa, 1051/408, Bairro Cidade Baixa, Porto Alegre/RS, Brazil (e-mail: denisemanica@gmail.com).
\end{abstract}

Int Arch Otorhinolaryngol 2013;17:407-408.
Abstract
Keywords
- dermoid cyst
- infant
- newborn
- nasopharynx
- nasopharyngeal neoplasms
- respiratory distress syndrome

The dermoid is a rare and benign malformation of bigerminal origin. It is seen frequently in the neonatal period, and the clinical presentation depends on the site and on the size of the lesion, and the classical clinical picture is of a tumor derived from the naso- or oropharynx, leading to respiratory distress and/or feeding disorders. A female newborn was born cyanotic requiring intubation. The oroscopy revealed a large smooth mass. Transoral surgery was performed with successful extubation. Seven months after, there are no signs of recurrence of the lesion. This report adds another case of this rare pathology that can be life-threatening condition in the neonate. It emphasizes the importance of dermoid in the differential diagnosis of the naso- or oropharyngeal lesions, especially in neonates.

\section{Introduction}

The dermoid, or hairy polyp, is a rare and benign malformation of bigerminal origin with both ectodermal and mesodermal elements. It is seen frequently in the neonatal period, but occasionally it can be found in older children. ${ }^{1-3}$ The clinical presentation depends on the site and on the size of the lesions, and the classical clinical picture is of a tumor derived from the naso- or oropharynx, leading to respiratory distress and/or feeding disorders. This case report presents one case of dermoid causing respiratory difficulties at birth.

\section{Case Report}

A 3,050-g female patient was born by spontaneous vaginal delivery at 35 weeks' gestation. The child was born hypotonic and cyanotic, with Apgar scores 5, 6, and 7. The child required intubation and remained in mechanical ventilation. The mother, 38 years old, attended nine prenatal consultations without gestational problems and with negative serology results, except toxoplasmosis IgG reagent.

On physical examination, cardiac, pulmonary, and abdominal examinations were normal. Oroscopy revealed a large smooth mass ( - Fig. 1). On the flexible fiberoptic laryngosco- py, it could be seen that the lesion protruded from the left nasopharyngeal wall.

Magnetic resonance imaging showed an expansive lesion with signal intensity similar to fat tissue without significant contrast enhancement (-Fig. 2). Transoral surgery with complete excision of the lesion was performed. The histologic analysis described a polyp composed by adipose tissue, fibrosis, skeletal muscle, mature cartilage, and skin compatible with dermoid.

The child was extubated in the third day of surgery and remained without respiratory distress. Oral feeding was initiated after 1 week with good acceptance. Now this patient is 7 months old without any signs of recurrence of the lesion.

\section{Discussion}

The dermoid was first described by Brown-Kelly in $1918 .^{3}$ One hundred thirty-five cases were reviewed by Kelly et al in 1996. ${ }^{4}$ After this review, Yilmaz et al in 2012 reviewed 35 more cases, increasing the number of patients reported in the literature to 170 cases. $^{2}$

Dermoids are histologically composed by stratified keratinized epithelium with cutaneous structures like hair and received

November 4, 2012

accepted

December 28, 2012
Copyright ( 2013 by Thieme Publicações DOI http://dx.doi.org/ Ltda, Rio de Janeiro, Brazil $10.1055 / \mathrm{s}-0033-1351677$. ISSN 1809-9777. 


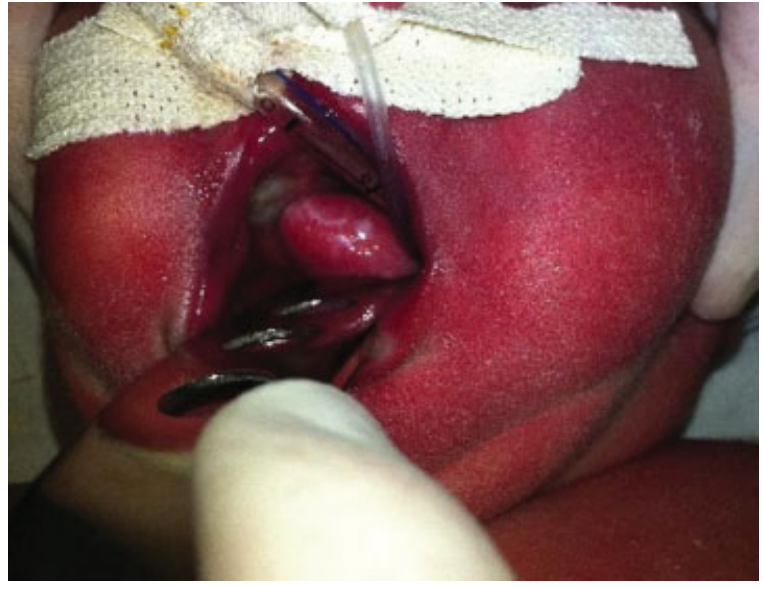

Fig. 1 Transoral view of the lesion.

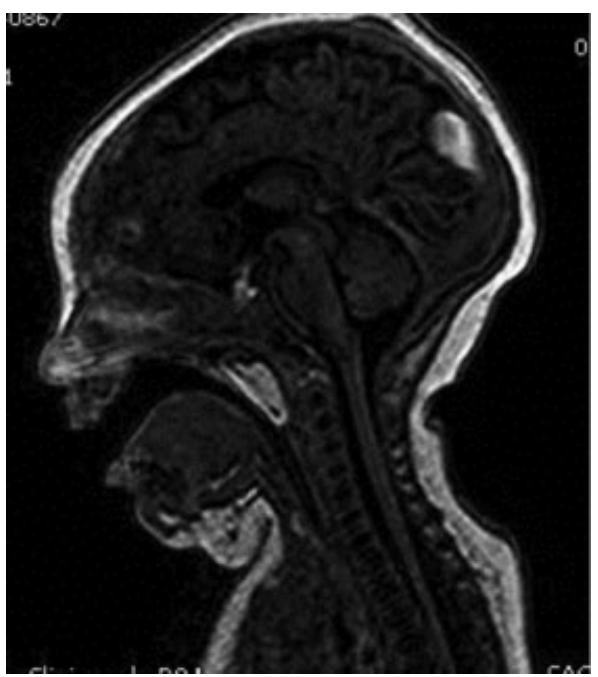

Fig. 2 Sagittal magnetic resonance image demonstrating soft tissue lesion in nasopharynx.

sebaceous and sudoriparous glands. ${ }^{2,3}$ The classical clinical presentation is the visualization of a pedunculated lesion in the pharynx, ${ }^{5}$ as seen with the patient described here. The respiratory distress can be dramatic with asphyxia at birth or can be subtle with intermittent obstruction. Polyhydramnios during the pregnancy can be seen because of the obstruction of the swallowing mechanism. There can also be vomits, feeding disorders, hemoptysis, eustachian tube disorder, and increased unilateral nasal secretion. ${ }^{6}$

Dermoids occur six times more frequently in females. The most common location is in the naso- or oropharynx, in the left side. The reason for predilection for females and left side, as in the described case, is unknown. ${ }^{2,3}$

Dermoids are rarely associated with other congenital abnormalities such as cleft palate, absent uvula, auricle deformities, ankyloglossia, facial hemihypotrophy, atresia of left carotid artery, and osteopetrosis. However, it is important to investigate other congenital malformations. The present case did not present any such associated problems.

The differential diagnosis of a neonatal nasopharyngeal mass includes teratoma (different than teratoma in that the dermoid does not have endodermal structures), encephalocele, intranasal glioma, neuroblastoma, hemangioma, and thyroglossal or lingual cyst. The first diagnostic procedure is the flexible fiberoptic laryngoscopy that allows establishment of the size, origin, and extension of the mass. Radiologic studies like computed tomography and magnetic resonance imaging are essential for the surgical planning. ${ }^{3}$

The initial management focuses on maintaining a secure airway, frequently with endotracheal intubation. The definitive treatment is surgical excision. The histologic analysis is important for the prognosis and follow-up. The dermoid prognosis is excellent, and there were no cases of recurrence or malignant transformation. ${ }^{2,3}$

\section{Conclusion}

This report adds another case of this rare pathology that can be a life-threatening condition in the neonate. It emphasizes the importance of dermoid in the differential diagnosis of the naso- or oropharyngeal lesions, especially in neonates.

\section{References}

1 Chakravarti A, Vishwakarma SK, Arora VK, Singh I. Dermoid (hairy polyp) of the nasopharynx. Indian J Pediatr 1998;65:473-476

2 Yilmaz M, Ibrahimov M, Ozturk O, Karaman E, Aslan M. Congenital hairy polyp of the soft palate. Int J Pediatr Otorhinolaryngol 2012;76:5-8

3 Kalcioglu MT, Can S, Aydin NE. Unusual case of soft palate hairy polyp causing airway obstruction and review of the literature. J Pediatr Surg 2010;45(12):e5-e8

4 Kelly A, Bough ID Jr, Luft JD, Conard K, Reilly JS, Tuttle D. Hairy polyp of the oropharynx: case report and literature review. J Pediatr Surg 1996;31:704-706

5 Roh JL. Transoral endoscopic resection of a nasopharyngeal hairy polyp. Int J Pediatr Otorhinolaryngol 2004;68:1087-1090

6 Agrawal N, Kanabar D, Morrison GA. Combined transoral and nasendoscopic resection of an eustachian tube hairy polyp causing neonatal respiratory distress. Am J Otolaryngol 2009;30:343-346 